\title{
D-Branes on Calabi-Yau Manifolds
}

\author{
Michael R. Douglas
}

\begin{abstract}
We give an overview of recent work on Dirichlet branes on CalabiYau threefolds which makes contact with Kontsevich's homological mirror symmetry proposal, proposes a new definition of stability which is appropriate in string theory, and provides concrete quiver categories equivalent to certain categories of branes on CY.
\end{abstract}

\section{Introduction}

Dirichlet branes, discovered by Dai, Leigh and Polchinski in 1989, have been a central part of the dramatic progress in superstring theory of recent years. They are the simplest solitons in string theory; the basic definition of Dirichlet brane (D-brane) is that it is an allowed boundary condition for a string. [45]

Although they play many physical roles, from a mathematical point of view their most salient feature is that they provide the natural context in which YangMills theory is embedded in string theory. This has allowed physicists to rederive and better understand some of the most beautiful mathematical constructions which have been discovered in this area, such as the ADHM construction of instantons and Nahm's construction of monopoles.

D-branes on Calabi-Yau (CY) manifolds have been the focus of a number of recent works (see $[15,18]$ for additional references). Early on it was realized that this theory had a close connection with mirror symmetry and especially Kontsevich's homological mirror symmetry proposal [36], which interprets mirror symmetry as an equivalence between the derived category of coherent sheaves (naturally associated to Yang-Mills on Kähler manifolds) and a derived category proposed by Fukaya, associated to isotopy classes of Lagrangian submanifolds. These two classes of objects (usually called "B" and "A"-type branes respectively) correspond to the two classes of supersymmetry preserving (or BPS) branes on a CY, and thus the physical understanding of mirror symmetry indeed should imply such an equivalence.

In physical terms, Kontsevich's proposal addresses only the structure of topologically twisted open string theory (we explain this below), while one expects more structure to appear in discussing the full open string theory which governs the physics of D-branes. 
One approach to this extra structure is to study the additional conditions on the D-brane world-volumes which follow from space-time supersymmetry. In the large volume limit, these two conditions are the hermitian Yang-Mills equations (for B branes) and the special Lagrangian condition (for A branes). In this context, Strominger, Yau and Zaslow formulated mirror symmetry as the statement that the mirror $W$ to a CY $M$ could be obtained as the moduli space of a particular A brane mirror to the B brane which is the point on $M$. [52] This brane is expected to come in families which give a $T^{3}$ fibration structure to $M$, a claim which has been proven in many cases by Gross and by Ruan. [59, 27, 49, 41]

Although this approach clearly captures some essential part of the truth, it should be realized that the specific additional conditions which were used, the hermitian Yang-Mills and special Lagrangian conditions, are not believed to be the correct physical conditions except in the large volume limit. This is related to the point that in string theory, CY manifolds are not really Ricci-flat manifolds except in the large volume limit; the Einstein equations are known to be corrected. Indeed, the stringy version of CY Kähler moduli space, which is best thought of as the complex structure moduli space of the mirror, looks quite different from the conventional geometric version.

The correct replacements for either Ricci-flatness or the supersymmetry conditions on D-branes are not known at this writing, but some things are known, which we summarize here. In particular, one has strong reasons to believe that the metric defined by the SYZ construction (which is the "D0-metric" of [14] on the mirror manifold) is not Ricci-flat.

Having cast doubt on the usual mathematical starting point for the discussion of D-branes, we are obliged to say something about what replaces it. Now the fundamental physical definition is quite clear - a D-brane is a boundary condition in the world-sheet conformal field theory (CFT); the A and B branes each correspond to boundary conditions preserving a particular half of world-sheet supersymmetry.

This definition has some conceptual advantages; notably, as in the discussion of Greene and Plesser [25], mirror symmetry is manifest. The problem with it is that it is rather hard to make precise contact with geometry except in the large volume limit. However, a number of general results have been obtained, which we outline. In keeping with the CFT framework, we freely use mirror symmetry in our considerations, while regarding the precise connection with geometry as following from subsequent considerations which are not yet completely understood.

An example of a geometrical statement which is on a sound footing in string theory is the statement that the holomorphic properties of B branes are independent of Kähler moduli, and thus are "the same" as in the large volume limit $[4,14]$. The precise meaning of "same" turns out to be subtle however and this is the point where the derived category must enter the discussion. A recent development is a precise contact with the graded categories which appear in Kontsevich's proposal, which shows that (if we consider B branes), as the Kähler moduli are varied, the 
gradings undergo a "flow" determined by the variation of certain phases associated with the branes (in A brane language, the phase appearing in the special Lagrangian condition). [17, 19]

This puts us in a position to explain perhaps the simplest physical structure carried by D-branes which is not already implicit in [36]. This is the concept of "marginal stability" which governs the possible variation of the spectrum of BPS branes. This concept reduces to variation of $\mu$-stability for $\mathrm{B}$ branes in the large volume limit and based on this analogy, the work of Joyce on such transitions for A branes [34], and many other considerations, a deformation of stability called $\Pi$-stability was proposed in [16] which governs the spectrum of BPS branes on arbitrary CY's in string theory. This proposal has undergone a number of tests; we also briefly describe work in progress which attempts to complete the proposal by associating natural $t$-structures to regions in Kähler moduli space.

Another front on which physical considerations have made progress is in the explicit construction of these BPS branes and their moduli spaces. A particularly simple class of constructions are the orbifold constructions. These can be exactly solved for orbifolds of flat space, and provide a physical context which contains the structures found both in orbifold resolution by quiver varieties [37] and in the study of the generalized McKay correspondence [3, 32, 48], including generalizations of Beilinson's construction of holomorphic bundles on $\mathbf{P}^{n}$. [1]

More recently, it has been found that results from the study of explicit Gepner model boundary states [47] can be rederived starting with a "LandauGinzburg orbifold theory." [18] Geometrically, this uses an embedding of the CY in a weighted projective space, and describes bundles on CY by using the generalized McKay approach to describe bundles on the ambient space, and then restricting. One new point which emerges is that the physical construction naturally contains moduli which appear only on restriction. Another striking result from this approach is an explicit prediction for the connection formula which relates the natural bases of periods at the large volume and Gepner points.

\section{Background on CFT on Calabi-Yau}

The most general definition of a Dirichlet brane in weakly coupled type II string theory is that it is a conformal boundary condition in the world-sheet CFT. This definition is discussed at this level of generality in [45]. It is quite analogous to the discussion of branes in a geometrical framework as calibrated submanifolds carrying appropriate vector bundles in the sense that they are auxiliary objects; one requires some description of the ambient space (the CY for us) to get started on their definition.

In the most general case, where we take as the "ambient space" an abstract CFT defined in terms of a Hilbert space, Hamiltonian and operator product coefficients, our starting point is a priori non-geometric: it does not come with any local coordinates or other conventional geometric data. On general grounds, the 
CFT's which are easy to describe in this abstract way are "highly stringy" - the conventional equations of physics such as the Einstein and Yang-Mills equations will not be valid (except in special cases with high symmetry) but will be drastically modified in string theory (we will say more about this below). In some cases, such as the Gepner models, we can argue that they are still connected to solutions of Einstein's equations by varying parameters (this is the "large volume limit"), and the interesting question is then what aspects of this conventional geometrical interpretation survive the stringy modifications and how do other aspects change. However, there are other cases such as asymmetric orbifolds [42] where we have no such large volume limit or other geometrical interpretation at present. In these cases, the abstract definition of D-brane makes sense, but the identification of D-branes with cycles carrying bundles on some space cannot even get started.

Although general, these remarks are intended to make the point that many of the statements about branes which are taken as definition or otherwise manifest in the mathematical treatments, actually require proof or at least justification in the physical discussion starting from string theory. While we don't want to belabor this point, it should be kept in mind.

We now specialize to the particular case of string compactification on a Calabi-Yau threefold, for which the CFT is a $(2,2)$ superconformal field theory with $\hat{c}=3$. These have been discussed in $[7,9,41,55]$ and elsewhere. The notation $(2,2)$ means there are two independent $N=2$ superconformal algebras describing left and right-moving excitations on the string world-sheet. Each $N=2$ algebra has two supercharges $G^{+}$and $G^{-}$which anticommute to produce the Hamiltonian and a $U(1)$ charge $J$, which provides a grading on the Hilbert space.

A distinguishing feature of the $N=2$ algebra is that it admits a topological twisting, a redefinition of the generators after which one of the supercharges can be used as a "BRST charge," a differential $Q$ which squares to zero. The cohomology of the sum $Q_{L}+Q_{R}$ of these operators in the two $N=2$ algebras is finite dimensional and is the Hilbert space of the topologically twisted theory. This Hilbert space also parameterizes a space of "operators" in the theory which correspond to linearized deformations of the theory; these always turn out to integrable in the $(2,2)$ case so these theories come in families with locally smooth moduli spaces.

Physicists are particularly interested in these models not because they admit topological twisting but because they lead to string theories with space-time supersymmetry. Seeing this requires discussing a bit more structure, namely the "bosonized $U(1) . "$ The main point here is that there are natural "spectral flow" operators denoted $e^{i \hat{c} \phi / 2}$ (on left and right) which provide automorphisms between sectors of different $U(1)$ charge $q$ and $q+\hat{c} / 2$. Sectors with integral (resp. halfintegral) $U(1)$ charge correspond to space-time bosons (fermions) and this oneto-one relation guarantees space-time supersymmetry. The topologically twisted world-sheet theories are important in this context mainly because their observables correspond to certain distinguished amplitudes in this space-time supersymmetric theory. 
The case of threefolds with $\hat{c}=3$ is further distinguished in that the correlator of three operators (the "Yukawa coupling") is always well-defined; one can further show that such correlators are the third derivatives of a function on moduli space $F$ known in the physics literature as the " $N=2$ prepotential" and in the math literature as the "Gromov-Witten potential." We will discuss the physical interpretation of this potential below.

One made a choice in this construction of whether to identify $G^{+}$or $G^{-}$as the BRST operator $Q$; it turns out that only the relative choice between the two $N=2$ algebras matters in the final result. This choice distinguishes "A" and "B" topologically twisted theories, which although they arise from the same CFT, need have little else in common. On the level of abstract CFT, one can also exchange $G^{+}$and $G^{-}$in one of the $N=2$ algebras to define a "new" CFT, all of whose physical predictions would be the same, but in which the A and B twisted theories would be exchanged. Thus there is no a priori difference between A and B, and this is the sense in which mirror symmetry is manifest in CFT.

This formalism arises from several more concrete constructions. Contact with geometry and the large volume limit is most direct in what is called the "nonlinear sigma model" definition. This starts from a Calabi-Yau threefold with Ricci-flat metric $g^{(0)}$, and maps of the string world-sheet into this "target space." The Ricciflat metric is determined by a choice of complex structure (let the moduli space of these be $M C(M)$ ) and complexified Kähler class (moduli space $M K_{0}(M)$ ); physical arguments strongly suggest that this produces a $(2,2)$ CFT determined by these moduli. The two gradings provided by left and right $U(1)$ charge essentially correspond to the bigrading of Dolbeault cohomology, and the B and A twisted theories as above correspond respectively to a theory describing variation of Hodge structure (the Yukawa couplings are the second derivatives of the periods of the holomorphic three-form) and to the "quantum cohomology theory" whose prepotential generates the Gromov-Witten invariants.

Mirror symmetry equates the A model on $M$, which sees only $M K(M)$, with the B model on its mirror $W$, which only sees $M C(W)$. In particular, the complexified Kähler cone $M K_{0}(M)$ is only a large volume approximation to the "true" stringy Kähler moduli space $M K(M)$, which is best defined simply as $M C(W)$. Much work has been done on the physical interpretation of this point. [24] Since there is such a close connection between these topologically twisted theories and the complex geometry, the claim that these originate from physical CFT's for which mirror symmetry is manifest places this formulation of mirror symmetry essentially beyond doubt; various explicit arguments have confirmed this. [41]

Beyond these results which can be convincingly justified in the topologically twisted string theory, what one actually has in this definition is a way to compute general observables as a series expansion in powers of the curvature of the metric $g^{(0)}$ multiplied by a distinguished scale of length $l_{s}$, the "string length." One also has prescriptions for adding "instanton" corrections associated to non-trivial holomorphic maps from world-sheets of various genus into the CY. It should be 
realized that this expansion by itself does not provide a very convincing argument for existence of these models on finite size CY, because such series expansions often do not converge. However, given evidence from other directions that models do indeed exist, we can interpret them as valid descriptions at least of the asymptotics of these observables for large CY's. (Whenever we talk about "large" CY's in the following, we simply mean the case in which the leading terms are good approximations.)

Perhaps the most basic observation one can make from these results is that a CY is actually not Ricci flat in string theory [26]. One traditionally derives Ricciflatness as the condition for "zero beta function" required for conformal invariance; however this is just the leading term and one sees the next correction at order $l_{s}^{6}$; the true condition takes the form

$$
0=\frac{\partial}{\partial \mu} g_{i j}^{\beta}=R_{i j}+l_{s}^{6}\left(R^{(4)}\right)_{i j}+\ldots
$$

with the term $R^{(4)}$ given in [26], and a higher order term shown to be non-zero in [33]. It has also been shown that the corrections are such that a solution can be found at each order in the series [43].

A conceptual argument that Ricci-flatness is not to be expected is simply that the structure of the Kähler moduli space predicted by mirror symmetry is quite different from the geometric Kähler cone or a naive complexification of this, and we would expect the "true" stringy metric to depend naturally on this true moduli space; the space of Ricci-flat metrics does not.

However, it is difficult to go further with this definition, simply because neither the equation (1) nor the metric $g^{\beta}$ it describes are canonically defined; the theory of renormalization which led to it does not distinguish in any convincing way between $g^{\beta}$ and any other metric $g^{\beta^{\prime}}$ which would be produced by an arbitrary local functional redefinition $g \rightarrow g+\alpha_{1} R+\alpha_{2} R^{2}+\ldots$.

The study of D-branes will provide a better definition of the metric, which we discuss in the next section. Let us turn now to alternatives to the non-linear sigma model. The most famous of these is the Gepner model, also described in the references above. This starts off from completely non-geometric data, but is believed to be equivalent to the "analytic continuation" of the CY non-linear sigma model to a specific highly stringy point in the true Kähler moduli space. The fact that such models do exist and are described by the $N=2$ formalism is our best reason to believe that the series expansions described earlier can indeed be summed in some way to define the models under discussion.

The best understanding we have at present of how the Gepner model is connected to the geometric picture comes through Witten's "linear sigma model" definition ([56]; see [23, 30] for work on D-branes in this framework). One can think of this as defining the CY through a specific embedding in $\mathbf{C}^{6}$. This embedding is described in two steps. First, one constructs a weighted projective space as a symplectic quotient of $\mathbf{C}^{6}$ by a $U(1)$ action, in coordinates $z^{i} \rightarrow e^{i w_{i} \theta} z^{i}$ for chosen 
weights $w_{i}$. One then defines the $\mathrm{CY}$ as a complex hypersurface in this ambient space. (One can similarly get more general toric varieties, etc.)

The symplectic quotient is the ordinary quotient by $U(1)$ of the stable points in the preimage $\pi^{-1}(0)$ of a moment map $\pi: \mathbf{C}^{6} \rightarrow \mathbf{R}$ defined by

$$
\left\{z^{i}\right\} \rightarrow-\mu+\sum w_{i}\left|z^{i}\right|^{2}
$$

To get weighted projective space with weights $w_{i}$, one takes $w_{i} \geq 0$ for $1 \leq$ $i \leq 5, w_{6}$ such that $\sum_{i} w_{i}=0$, and $\mu>0$. Actually, the quotient is a line bundle $O_{W P}\left(w_{6}\right)$ over the weighted projective space; one then imposes the complex equations grad $W=0$ with $W=z^{6} f\left(z^{i}\right)$, which reduce to $z^{6}=0$ and $f\left(z^{i}\right)=0$ within this zero section.

The important point is now that one can argue that varying the true Kähler parameter to the Gepner point corresponds to an analytic continuation to the "Landau-Ginzburg phase," the limit $\mu<<0$. Here the symplectic quotient is rather different: it is the orbifold $\mathbf{C}^{5} / \mathbf{Z}_{-w_{5}}$, and the equations grad $W=0$ now constrain the coordinates to $z^{i}=0$ for $1 \leq i \leq 5$. Although on the surface this looks quite different from the original nonlinear sigma model, since the orbifold is birational to the original line bundle, from the point of view of complex geometry the two phases are not so different, and further physical considerations bear this out, leading to a moduli space $M K$ covering both phases with only isolated singularities, and no immediately evident distinction between the phases.

This discussion motivates the idea that a good starting point for defining CY is orbifold resolution, and indeed an even simpler class of CY's can be defined this way, as the resolution of orbifolds $\mathbf{C}^{3} / \Gamma$ with $\Gamma$ a discrete subgroup of $S U(3)$. This produces non-compact CY's, which have also been much studied as local models of singularities in CY's, with the general conclusion being that mirror symmetry and almost all of the qualitative features of CY physics are visible in these simpler examples. The simplest case is $\mathbf{C}^{3} / \mathbf{Z}_{3}$, studied in $[12,13,17]$.

\section{Dirichlet branes and stability}

The coarsest classification of boundary conditions in CFT is according to the portion of the superconformal algebra they preserve. One way to specify a boundary condition is in terms of a linear transformation implementing the "reflection" from left-moving to right-moving excitations; this transformation must contain an automorphism of the entire symmetry algebra and thus we need to know these.

In the $(2,2)$ context, there are two basic automorphisms one can use to relate left and right moving algebras. [44] The trivial choice $G_{L}^{ \pm}=G_{R}^{ \pm}$and $J_{L}=J_{R}$ is referred to as a "B-type" boundary condition as it is compatible with the B topological twisting. ${ }^{1}$ Such boundary conditions can be obtained in the non-linear sigma model by fixing the boundary to sit on a holomorphic submanifold of the CY carrying a holomorphic bundle. The other basic choice is $G_{L}^{ \pm}=G_{R}^{\mp}$ and

\footnotetext{
${ }^{1}$ This twisting is defined in open string conventions.
} 
$J_{L}=-J_{R}$ or "A-type," which is obtained by fixing the boundary to sit on a Lagrangian submanifold, carrying a flat bundle.

This is all the data which is required to specify a boundary condition in the topologically twisted open string theory [57], and in this context mirror symmetry can be interpreted as a one-to-one equivalence between the two classes of boundary conditions and all topological correlators. This is the physical background behind Kontsevich's proposal; however there is much more to say about its physical underpinnings and meaning which has only been addressed recently.

From a physical point of view, the most basic question is what physical observables could one compute if one knew something about the derived category of coherent sheaves or its mirror. This question is not entirely trivial as the categorical framework is quite different from the usual physics language, which assumes that the objects involved come in moduli spaces with explicit coordinatizations, etc.

There are various physical realizations of the BPS branes we are talking about, depending on what string theory we use and how many flat dimensions the brane extends in. All are essentially identical in terms of the underlying string theory; the primary distinction is between those which can be treated purely classically (so, objects are solutions of equations of motion derived from some action), and those in which quantum effects are taken into account (so objects are zero energy wave functions or typically harmonic forms on the classical moduli space). In any case, they will have half of the supersymmetry available in the original type II theory on CY, because in that theory left and right-movers led to independent supersymmetries, which are now related by the boundary conditions.

Two realizations seem to be particularly useful to keep in mind. In the first, the physical picture is of open strings free to move in the $4=10-6$ dimensions not taken up by the Calabi-Yau; this leads to a world-volume theory with $N=1$ supersymmetry in 4 dimensions, which we treat classically. These theories are gauge theories and the basic specification of such a theory is a choice of gauge group (a semi-simple Lie group) $G$, a Kähler manifold $X$ of "chiral matter" configurations admitting a $G$-action by isometries, a $G$-invariant holomorphic function $W: X \rightarrow$ C called "superpotential," and finally moment maps for the $G$ action.

Although all of this data is physical, for the primary question of finding the BPS branes and their moduli space (as a complex variety), the data only gets used in the following way: a configuration of the brane system is a solution of $\operatorname{grad} W=0$ in the symplectic quotient $X / / G$. We refer to the set of these as the moduli space of brane configurations. Since quotient commutes with restriction, one can also think of this as the symplectic $G$-quotient of the variety grad $W=0$.

The second realization takes the branes to be particles in the extra 4 dimensions. This description is related to the first as follows: given a moduli space of simple brane configurations (i.e. with endomorphism group $U(1)$ ); one identifies particles with zero energy states in a supersymmetric quantum mechanics on this moduli space. By standard arguments, these will be cohomology classes. (If the moduli space is singular, it turns out that the quotient/restriction definition gives 
it a natural embedding in a non-singular space, which can be used to make the definition.) These are the "BPS branes" of $[29,22]$ etc.

From now on we will consider only the first realization of brane, but use the second to define what we will call "BPS central charge" and explain the concept of marginal stability. It can be shown [9] that in an $N=2$ supersymmetric theory (such as type II on CY), the mass of a particle (in the usual space-time sense) satisfies a bound

$$
\begin{array}{rc}
M & \geq|Z(Q)| ; \\
Z(Q)=q_{i} z^{i}+m^{i} \frac{\partial F}{\partial z_{i}}=\int_{\Sigma} \Omega
\end{array}
$$

with equality attained only for BPS particles. The quantity $Z(Q)$ is the "BPS central charge" and depends only on the "charge" or topological type of the brane and on the CY moduli. It does not depend on the particular point or cohomology class in the moduli space of brane configurations.

For A branes, $Z(Q)$ is the integral of the holomorphic three-form over the the Lagrangian submanifold, and thus depends only on the homology class of the submanifold and the moduli $M C$. The BPS bound is the same as that coming from the calibrated geometry of special Lagrangian submanifolds. [28]

For B branes, $Z(Q)$ is defined in terms of the prepotential $F$, a function on $M K(M)$, usually computed by invoking mirror symmetry, and depends on the $\mathrm{K}$-theory class of the brane and the moduli $M K$. From now on, when we speak of "CY moduli space" we mean the part $(M C$ or $M K)$ which appears in these considerations, and "brane charge" means the homology or K-theory class.

The most basic physical role of this prepotential is its appearance in (2). Implicit in this setup is a flat (Gauss-Manin) connection on the CY moduli space, related to the choice of a fixed basis on homology. This allows transporting a BPS brane between points in moduli space and following the variation of its central charge.

Naively, this transport allows us to identify the complete spectrum (list) of BPS branes at each point in moduli space. However, this is not true, because of the existence of "lines of marginal stability" on which the spectrum changes. There is a strong constraint on these changes: a brane with charge $Q$ can undergo a decay (and thus disappear from the spectrum) into constituents with charges $Q_{i}$ (satisfying $Q=\sum Q_{i}$ ) only if the phases

$$
\varphi\left(Q_{i}\right) \equiv \frac{1}{\pi} \operatorname{Im} \log Z\left(Q_{i}\right)
$$

are equal. (The normalization factor $1 / \pi$ will be explained later.) This follows from energy conservation, which implies that $M \geq \sum M_{i}$, and (2), and no other assumptions. We will return to this point; for now we stress that the spectrum of BPS branes does change with moduli, in both realizations. In the first realization, it will turn out that this is the direct generalization of "wall crossing" phenomena related to the behavior of $\mu$-stability under variations of Kähler class. 
Once we know that moduli spaces of brane configurations exist, of course it would be quite interesting to know more about them and in particular to find their Kähler metrics. In particular, the problem of how to give a canonical definition of the metric of a CY in string theory has a natural answer in this context: it is just the moduli space of the "D0-brane," the B brane which embeds in a point. [14] This presupposes that such a brane exists and has a moduli space which is the CY, which although manifest at large volume cannot be taken for granted on a string-scale CY. Furthermore, there might not be a unique candidate; one can choose one by using the flat connection of Kähler moduli space to carry the large volume D0-brane along some path to the point of interest, a prescription which in general is path dependent.

Anyways, assuming that such a D0-brane exists, we stress that for each point in the true Kähler moduli space (the complex structure moduli space of the mirror), there exists a canonical Kähler metric on the CY, which away from the large volume limit is not expected to be Ricci flat (we will mention some explicit results bearing this out below [13]). This metric can also be thought of as the metric on the moduli space of the D3-brane wrapped on the $T^{3}$ fibration of SYZ mirror symmetry; again from this point of view, except in special limits, it has no reason to be Ricci flat. (In the explicit computations of [52], this would come about after instanton corrections.) It is not even clear that it has the same Kähler class as the original metric; indeed once one goes from $M K_{0}(M)$ to $M K(M)$ one loses any clear sense of what this would mean.

This metric or even the question of what equations determine it seems rather inaccessible at present and we return to the question of what Kontsevich's ideas should help physicists to compute. In some sense, the answer is the space $X$, group $G$ and superpotential $W$; however it has taken some time for physicists to recognize any of this data in Kontsevich and Fukaya's rather abstract derived categories, and indeed this relation has not been completely spelled out in the literature. The most basic point is simply that brane configurations can naturally be thought of as objects in a category whose morphisms are the linearized variations of the gauge theory data described above (tangent vectors to a point in $X$ modulo gauge directions) associated to the system which is the direct sum of two brane subsystems. The superpotential enters because such variations typically correspond to obstructed deformations; the non-trivial point seems to be that in cases associated to Calabi-Yau threefolds this obstruction theory can always be summarized in terms of gradients of a potential. This point has been recognized in numerous special cases - for example, the functional $W$ for holomorphic bundles is the holomorphic Chern-Simons functional, and for holomorphic curves is the functional proposed in $[10,58]$ - but a mathematical argument as simple and general as the physical argument based on $N=1$ supersymmetry does not seem to have appeared in the literature.

Having seen what Kontsevich's framework can describe, we also can see in this language what it does not describe: namely, the details of the symplectic quotient which are determined by the specific moment maps. In particular, not 
all holomorphic objects (solutions of grad $W=0$ ) correspond to points in this quotient; only stable objects do (in the GIT sense, which depends on the specific moment maps). Thus, the question of characterizing stable BPS branes (in the physical sense) could be answered by proposing the mathematical stability condition which they satisfy. One can show by physical arguments that just as the derived category of coherent sheaves does not depend on the Kähler structure of the CY, the moment maps should only depend on the Kähler structure and not on the complex structure. [19]

On large CY's, branes correspond to bundles satisfying the hermitian YangMills equations, so the stability condition is just the one given by the Donaldson and Uhlenbeck-Yau theorems, which is slope or $\mu$-stability: a bundle $E$ (or coherent sheaf) is $\mu$-stable if, for all subsheaves $E^{\prime}$, one has $\mu\left(E^{\prime}\right)<\mu(E)$ with the slope $\mu(E)=c_{1}(E) / \operatorname{rank}(E)$. This condition depends on both Kähler and complex structure but in a clearly separated way: $\mu$ depends on Kähler structure, and the subobject relation on complex structure. This will be the prototype for our stringy stability condition.

A first step towards such a condition is to consider a deformed hermitian Yang-Mills equation derived from string theory by Marino et. al. [40] as the condition for unbroken supersymmetry from the "Born-Infeld action." Their equation is a deformation of the Yang-Mills action which involves higher powers of the Yang-Mills curvature $F$, and takes the form

$$
0=\mu_{d, \theta} \equiv \operatorname{Im} e^{i \theta} \operatorname{Tr}\left(\omega+i l_{s}^{2} F\right)^{d}
$$

on a $d$-fold with Kähler form $\omega$. Considerations of geometric invariant theory in $[38,53]$ show that such an equation will have a solution for " $\mu_{\theta}(E)$-stable" bundles; i.e. a stability condition with $\mu_{\theta} \equiv \int \mu_{d, \theta}$ playing the role of the slope.

Although very explicit, this equation only takes into account power-like corrections in $l_{s}$, while it is known that further instanton corrections are present. Related to this, it is not clear which Kähler form one should take for $\omega$ (the Ricci-flat or some stringy version.) This point could also be addressed by D-brane considerations, but it is not clear that this is the best approach to take as one would prefer to have a condition which depends not on $\omega$ but on the point in $M K(M)$, i.e. on the complex structure of the mirror.

One can identify the precise quantity which generalizes slope by appealing to mirror symmetry to relate this question to work of Joyce on invariants counting special Lagrangian manifolds. [34] Among the many results of this work is an analysis of the local stability of a special Lagrangian manifold under variations of the complex structure; this is determined by an inequality involving (in physics language) the phase of the BPS central charge (3). This fits very well with the known physical considerations on marginal stability, and the sign of the inequality is new information from this point of view.

This brings us to the proposal of [16], that the BPS branes at a specific point in (true) Kähler moduli space are the "П-stable" branes, i.e. each brane based on a holomorphic object $E$ such that for all subobjects $E^{\prime}$ one has $\varphi\left(E^{\prime}\right)<$ 
$\varphi(E)$. Compared to Joyce's criterion, the main difference is to use morphisms and subobjects in the definition. There is also a conceptual difference: instead of working with special Lagrangians, we take the stability condition back to the $\mathrm{B}$ picture and apply it there. This has the great practical advantage that the definition of subobject is fairly clear in the B picture (though we will have to say more about this below) while computing the morphisms in the A picture is a harder and still not fully understood problem.

Besides the arguments we cited, in [16] the proposal is compared with more explicit results in the large volume and in orbifold limits (more below) and finds agreement. It was studied in the example of the $\mathbf{C}^{3} / \mathbf{Z}_{3}$ orbifold and its resolution in [17], about which more below, and seems to produce sensible results there. As we mentioned, on physical grounds it is impossible for the spectrum to change except on lines of marginal stability as defined earlier, and it is hard to come up with any competing proposal which satisfies this constraint. Having said this, the proposal has two ill-defined points. First, one must lift the phases from the interval $[0,2)$ (which is what (3) gives us) to $\mathbf{R}$. The geometry underlying this (in the A picture) is explained in [50]; in our work we have only postulated this lifting ad hoc. Second, it requires objects to live in an abelian category (as do all GIT notions of stability), but the only universal category associated to a Calabi-Yau seems to be the derived category used by Kontsevich, which is not abelian. The two points are connected and we will come back to them below.

For completeness, we should say that (in our opinion) the assumption that A branes are in fact special Lagrangian submanifolds is on less firm a footing than most of the other elements of the picture. The basic problem is the one that we mentioned, that the appropriate definition of the metric and Kähler form of the CY in string theory has not in fact been settled, but is almost certainly not the Ricci flat metric. One possibility is that one still has the special Lagrangian condition but with respect to a preferred non Ricci flat Kähler form, possibly the D0-metric described above. Some evidence for this idea can be found in the work of Leung, Yau and Zaslow [39] which shows that the special Lagrangian condition is related to the MMMS equation (4), in which the metric could be derived from D-brane considerations, by a Fourier-Mukai transform of the type which should describe T duality in string theory. At present however this is just a guess, and more physical work is needed to understand this point; for example it is clear that with some work a series expansion analogous to (1) could be found for the corrections to the special Lagrangian condition.

\section{Flow of gradings}

We now turn to a point which emerges quite clearly from physics and CFT but does not seem to have appeared in the mathematical discussion, namely a "flow of gradings" which is induced under variation of Kähler moduli (in the B picture) or complex structure (in the A picture). 
Consider the B branes for definiteness; in the large volume limit each brane corresponds to some stable coherent sheaf and thus for each pair of branes $E$ and $F$ we have graded morphisms $\operatorname{Ext}^{n}(E, F)$. One often denotes this space as $\operatorname{Hom}(E, F[n])$, a notation which finds its justification in homological algebra.

Suppose we now follow a general path in Kähler moduli space between two points $K$ and $L$. The new claim is that a morphism of degree $n$ at the starting point $K$ will undergo "flow of grading" determined by the phases (3): its degree at the point $L$ will be

$$
n^{\prime}=n+\varphi_{L}(F)-\varphi_{K}(F)-\varphi_{L}(E)+\varphi_{K}(E)
$$

(This is the reason for the $1 / \pi$ in (3), which first appeared in [46].) In particular, the gradings are $\mathbf{R}$-valued. This rule can be expressed more simply by saying that the grading of objects varies with $\varphi$, so that the starting $\operatorname{Hom}\left(E\left[\varphi_{K}(E)\right], F[n+\right.$ $\left.\left.\varphi_{K}(F)\right]\right)$ turns into $\operatorname{Hom}\left(E\left[\varphi_{L}(E)\right], F\left[n+\varphi_{L}(F)\right]\right)$.

In itself this is just a definition but it can be checked physically if we have more than one definition of the underlying CFT. As we discuss in the next section, this check has been made between large volume and orbifold points in various models. The phases are known from mirror symmetry results and flow by integers between these points, and the gradings of morphisms agree with these predictions.

The argument for the flow from CFT is quite simple. [19] It relies on the identification of a morphism with a "winding string in the bosonized $U(1)$," and has the following intuitive picture. One can think of each brane as having location $\varphi$ on a circle of circumference 2 (the bosonized $U(1))$ and a morphism as an open string stretched between the pair of branes; grading corresponds to length, so the flow is simply induced by motion of the branes on the circle.

Once we grant this point, we quickly see that no single abelian category (such as the category of coherent sheaves) could possibly describe the branes throughout Kähler moduli space. This is because a general path will cause gradings to flow below zero, and convert objects from even to odd grading, both of which lead to violations of the axioms of an abelian category. However, the derived category and its distinguished triangles still make sense with these flows. This is perhaps the fundamental reason why one is forced to the derived category in these considerations. Recent work of Seidel and Thomas and of Horja [51, 31] provides even more concrete motivation for this point; they show that the monodromies associated to general paths in true Kähler moduli space act naturally on the derived category, not the category of coherent sheaves.

One does not have a notion of subobject in the derived category and thus no GIT definition of stability can apply in this context. The most direct way out of this problem is to propose that each point in Kähler moduli space comes with a preferred abelian category. This could be defined by a choice of $t$-structure [2], which might be determined by the phases as well: one basically wants to keep all objects whose phases lie in the interval $(-1,0]$ and which are not involved in morphisms of negative degree. This point is presently under investigation. 


\section{Orbifolds}

The most accessible physical definitions of Calabi-Yau manifolds are the orbifolds $\mathbf{C}^{3} / \Gamma$ and the Landau-Ginzburg orbifolds described in the introduction. As it turns out, the basic constructions involved in defining D-branes in these theories are already fairly standard in mathematics and thus we can be relatively brief.

For B branes, one clearly wants to take some sort of $\Gamma$-equivariant bundles on $\mathbf{C}^{n}$, and this can be implemented in physical terms by starting with the gauge theory Lagrangian for branes in $\mathbf{C}^{n}$ and applying a quotient which acts simultaneously on $\mathbf{C}^{n}$ and in the gauge group. [11] This leads directly to the quiver theories which appear in the constructions of Kronheimer and subsequent work. [37] In particular, B branes are objects in a "McKay" quiver category, whose primitive objects (nodes) correspond to irreps of $\Gamma$, whose links correspond to terms in the tensor product with the representation defining the $\Gamma$ action on $\mathbf{C}^{n}$, and with specified quadratic relations.

The physical application requires $\Gamma \subset S U(n)$ and such orbifold singularities can often be resolved (always for $n \leq 3$ ) to smooth manifolds. This resolution is visible as the moduli space of a D0-brane and one can even compute the metric (actually, a controlled approximation in the small blow-up limit); for $\mathbf{C}^{3} / \Gamma$ one obtains explicit non-Ricci-flat metrics of the type discussed earlier. [13]

More generally, the question arises of how $\mathrm{B}$ branes as quiver objects or irreps of $\Gamma$ are related to the large volume definition of B branes as coherent sheaves. In recent mathematical work [3, 32, 48], a generalization of the McKay correspondence has been developed, which in particular provides a natural basis for $\mathrm{K}$ theory with compact support (thus supported on the exceptional divisor) labelled by irreps of $\Gamma$. The natural conjecture is that this is the physical correspondence we are looking for. [18]

This conjecture can be tested against results from mirror symmetry which determine the Gauss-Manin connection on Kähler moduli space and lead to a connection formula which relates the orbifold "charge" basis to the large volume basis. This gives an explicit expression for the Chern character of the bundle corresponding to each irrep, and this has been checked to agree with the conjecture for $\mathbf{C}^{3} / \mathbf{Z}_{3}$. [17] A somewhat more intricate version of this applies to the LandauGinzburg orbifolds; one uses the McKay correspondence to get the natural basis for the $\mathrm{K}$ theory of the weighted projective space, and then restricts this to the Calabi-Yau. Again one can compare with results from mirror symmetry and find agreement. [18]

These constructions are intimately related to and generalize Beilinson's construction of quiver categories from sheaves on $\mathbf{P}^{n}$ [1], and it is these categories of quiver representations which are compared to the category of coherent sheaves in the test of "flow of gradings" mentioned above. More generally, all this provides a fairly concrete relation between the problems of classifying coherent sheaves and of solving certain $N=1$ supersymmetric gauge theories. Although neither problem is easy in general, the second problem is not only more familiar to physicists 
but is a more appropriate language for the subsequent considerations than an explicit classification of sheaves would have been. It would be even more valuable if such constructions could be found for all sheaves, not just those which arise on restriction from the ambient space.

The first step in this generalization already appears in the physical theories it turns out that these include additional moduli which can be shown to correspond to linearized deformations of bundles which appear after restriction to the CY. [20] Presumably one could go beyond this linearized level by computing exact superpotentials; this may be possible using physical methods.

It would be quite interesting to know more about the variation of the spectrum of BPS branes with Kähler moduli. Some simple results of this type were found for $\mathbf{C}^{3} / \mathbf{Z}_{3}$ in [17]; for example it was shown that there are lines of marginal stability arbitrarily close to the large volume limit, by constructing bundles which were $\epsilon$ away from violating slope stability.

It would clearly be important to find a microscopic derivation of the stability condition, either from geometry (see [54] for work in this direction) or perhaps CFT or string field theory.

Our general conclusion has to be that Kontsevich's proposal, coming as it did before the study of D-branes, has turned out to be remarkably prescient. Although it has taken physicists some time to catch up, we are finally extending this picture to provide both more concrete pictures of branes on Calabi-Yau manifolds and new concepts which should be of interest to mathematicians.

We would like to thank D.-E. Diaconescu, B. Fiol and C. Römelsberger for enjoyable collaborations, and F. Bogomolov, M. Kontsevich, M. Marino, D. Morrison, A. Polishchuk, P. Seidel, and R. P. Thomas for valuable discussions. We thank R. P. Thomas for comments on the manuscript.

This work was supported by the Clay Mathematics Institute and by DOE grant DE-FG02-96ER40959.

\section{References}

[1] A. A. Beilinson, Coherent sheaves on $P^{n}$ and problems of linear algebra, Funct. Anal. Appl. 12 (1978) 214-216.

[2] A. A. Beilinson, Bernstein and P. Deligne, Faiseaux Pervers, Asterisque 100 (1982).

[3] T. Bridgeland, A. King, and M. Reid, Mukai implies McKay, math.AG/9908027.

[4] I. Brunner, M. R. Douglas, A. Lawrence, and C. Römelsberger, D-branes on the quintic, to appear in J. High Energy Physics, hep-th/9906200.

[5] P. Candelas, X. de la Ossa, A. Font, S. Katz, and D. R. Morrison, Mirror symmetry for two parameter models - I, Nucl. Phys. B416 (1994) 481, hep-th/9308083.

[6] P. Candelas, X. C. de la Ossa, P. S. Green, and L. Parkes, A pair of Calabi-Yau manifolds as an exactly soluble superconformal theory, Nucl. Phys. B359 (1991) 21.

[7] D. A. Cox and S. Katz, Mirror Symmetry and Algebraic Geometry, Mathematical Surveys 68, 1999, AMS. 
[8] J. Dai, R. G. Leigh and J. Polchinski, New Connections Between String Theories, Mod. Phys. Lett. A4, 2073 (1989).

[9] R. Dijkgraaf, Fields, Strings and Duality, in Les Houches LXIV, Symetries Quantiques, eds. A. Connes, K. Gawedzki and J. Zinn-Justin, Elsevier (1998).

[10] S. Donaldson and R. Thomas, Gauge Theory in Higher Dimensions, in The Geometric Universe; Science, Geometry, and the Work of Roger Penrose, Oxford University Press, 1998.

[11] M. R. Douglas and G. Moore, D-branes, Quivers, and ALE Instantons, hepth/9603167.

[12] M. R. Douglas, B. R. Greene, and D. R. Morrison, Orbifold resolution by D-branes, Nucl. Phys. B506 (1997) 84, hep-th/9704151.

[13] M. R. Douglas and B. R. Greene, Metrics on D-brane orbifolds, Adv. Theor. Math. Phys. 1, 184 (1998) hep-th/9707214.

[14] M. R. Douglas, Two Lectures on D-Geometry and Noncommutative Geometry, in Nonperturbative Aspects of Strings, Branes and Supersymmetry, World Scientific (1999), hep-th/9901146.

[15] M. R. Douglas, Topics in D-geometry, Class. Quant. Grav. 17 (2000) 1057, hepth/9910170.

[16] M. R. Douglas, B. Fiol, and C. Römelsberger, Stability and BPS branes, hepth/0002037.

[17] M. R. Douglas, B. Fiol, and C. Römelsberger, The spectrum of BPS branes on a noncompact Calabi-Yau, hep-th/0003263.

[18] M. R. Douglas and D.-E. Diaconescu, D-branes on Stringy Calabi-Yau Manifolds, hep-th/0006224.

[19] M. R. Douglas, D-branes, Categories and $N=1$ Supersymmetry, to appear.

[20] M. R. Douglas and D.-E. Diaconescu, to appear.

[21] B. Fiol and M. Marino, BPS states and algebras from quivers, JHEP 0007 (2000) 031, hep-th/0006189.

[22] R. Gopakumar and C. Vafa, M-Theory and Topological Strings-I, hep-th/9809187.

[23] S. Govindarajan, T. Jayaraman, and T. Sarkar, World sheet approaches to D-branes on supersymmetric cycles, hep-th/9907131.

[24] B. R. Greene, String theory on Calabi-Yau manifolds, hep-th/9702155.

[25] B. R. Greene and M. R. Plesser, Duality in Calabi-Yau Moduli Space, Nucl. Phys. B338 (1990) 15.

[26] M. T. Grisaru, A. E. van de Ven and D. Zanon, Two-Dimensional Supersymmetric Sigma Models On Ricci Flat Kahler Manifolds Are Not Finite, Nucl. Phys. B277 (1986), 388.

[27] M. Gross, Topological Mirror Symmetry, math.AG/9909015.

[28] F. Reese Harvey, Spinors and Calibrations, Academic Press (1990).

[29] J.A. Harvey and G. Moore, On the algebras of BPS states, Comm. Math. Phys. 197 489 (1998), hep-th/9609017.

[30] K. Hori, A. Iqbal, and C. Vafa, D-branes and mirror symmetry, hep-th/0005247. 
[31] R. P. Horja, Hypergeometric functions and mirror symmetry in toric varieties, math.AG/9912109.

[32] Y. Ito and H. Nakajima, McKay correspondence and Hilbert schemes in dimension three, math.AG/9803120.

[33] I. Jack, D. R. Jones and J. Panvel, Six loop divergences in the supersymmetric Kahler sigma model, Int. J. Mod. Phys. A8, 2591 (1993); hep-th/9311117.

[34] D. Joyce, On counting special Lagrangian homology 3-spheres, hep-th/9907013.

[35] S. Kachru, S. Katz, A. Lawrence and J. McGreevy, Mirror Symmetry for Open Strings, hep-th/0006047.

[36] M. Kontsevich, Homological Algebra of Mirror Symmetry, Proceedings of the 1994 ICM, alg-geom/9411018.

[37] P. B. Kronheimer, The construction of ALE spaces as hyper-Kähler quotients, J. Diff. Geom. 29 (1989) 665.

[38] N. C. Leung, Einstein Type Metrics and Stability on Vector Bundles, J. Diff. Geom. 45 (1997) 514.

[39] N. C. Leung, S.-T. Yau and E. Zaslow, From Special Lagrangian to Hermitian-YangMills via Fourier-Mukai Transform, math.DG/0005118.

[40] M. Marino, R. Minasian, G. Moore and A. Strominger, Nonlinear Instantons from Supersymmetric p-Branes, JHEP 0001 (2000) 005, hep-th/9911206.

[41] D. R. Morrison, Geometric Aspects of Mirror Symmetry, math.AG/0007090.

[42] K.S. Narain, M.H. Sarmadi and C. Vafa (Harvard U.), Asymmetric orbifolds, Nucl. Phys. B288 (1987), 551.

[43] D. Nemeschansky and A. Sen, Conformal Invariance Of Supersymmetric Sigma Models On Calabi-Yau Manifolds, Phys. Lett. B178 (1986), 365.

[44] H. Ooguri, Y. Oz and Z. Yin, D-branes on Calabi-Yau spaces and their mirrors, Nucl. Phys. B477, 407 (1996), hep-th/9606112.

[45] J. Polchinski, TASI Lectures on D-branes, hep-th/9611050.

[46] A. Polishchuk and E. Zaslow, Categorical Mirror Symmetry: The Elliptic Curve, Adv. Theor. Math. Phys. 2 (1998) 443-470, math.AG/9801119.

[47] A. Recknagel and V. Schomerus, D-branes in Gepner models, Nucl. Phys. B531 (1998) 185, hep-th/9712186.

[48] M. Reid, La correspondance de McKay, Séminaire Bourbaki (novembre 1999), no. 867, math.AG/9911165.

[49] W.-D. Ruan, Lagrangian torus fibration and mirror symmetry of Calabi-Yau hypersurface in toric variety, math.DG/0007028.

[50] P. Seidel, Graded Lagrangian submanifolds, math.SG/9903049.

[51] P. Seidel and R. P. Thomas, Braid group actions on derived categories of coherent sheaves, math.AG/0001043.

[52] A. Strominger, S.-T. Yau and E. Zaslow, Mirror Symmetry is T-Duality, Nucl.Phys. B479 (1996) 243-259, hep-th/9606040.

[53] R. P. Thomas, D-Branes and Mirror Symmetry, to appear in the proceedings of the 2000 Clay Mathematics Institute school on Mirror Symmetry.

[54] R. P. Thomas, to appear. 
[55] C. Voisin, Mirror Symmetry, SMF/AMS Texts 1, AMS 1999.

[56] E. Witten, Phases of $N=2$ theories in two dimensions, Nucl. Phys. B403 (1993) 159, hep-th/9301042.

[57] E. Witten, Chern-Simons gauge theory as a string theory, hep-th/9207094.

[58] E. Witten, Branes and the Dynamics of QCD, Nucl. Phys. B507 (1997) 658; hepth/9706109.

[59] I. Zharkov, Torus Fibrations of Calabi-Yau Hypersurfaces in Toric Varieties and Mirror Symmetry, math.AG/9806091.

Department of Physics and Astronomy,

Rutgers University,

Piscataway, NJ 08855-0849

and

I.H.E.S.,

Le Bois-Marie, Bures-sur-Yvette, 91440 France

E-mail address: mrd@physics.rutgers.edu 